\title{
Expression profile of mouse Mterfd2, a novel component of the mitochondrial transcription termination factor (MTERF) family
}

\author{
QianQian Xu, FengWei Zhang, HongJuan He, ShengQiang Xu, Kai Li, \\ ShanShan Liu, Yu Li and Qiong Wu* \\ State Key Laboratory of Urban Water Resource and Environment, Department of Life Science \\ and Engineering, Harbin Institute of Technology, No.92 West Da-zhi Street Harbin, \\ 150001, Heilongjiang, China
}

(Received 1 March 2011, accepted 15 August 2011)

\begin{abstract}
Mterfd2 is a component of mitochondria transcription termination factor (MTERF) family which belongs to the MTERF4 subfamily. In this report, we characterized the expression profile of mouse Mterfd2 during embryogenesis by in situ hybridization (ISH), quantitative real-time PCR (qRT-PCR) and northern blot. The whole mount ISH at E9.5, E10.5 and E11.5 showed that Mterfd2 was dynamically expressed in the brain. Besides, at E9.5 and E10.5 stages, Mterfd2 was persistently expressed in the lateral plate mesoderm and heart; at E10.5 and E11.5 stages, it showed an abundant expression in the limb buds. The tissue ISH of E13.5 and E15.5 suggested that Mterfd2 was ubiquitously expressed, and has the higher expression in the forebrain, diencephalon, midbrain, spinal cord, dorsal root ganglion, tongue, lung, liver and kidney. This ubiquitous expression profile in the late embryogenesis was further confirmed by qRT-PCR and northern blot at E12.5, E15.5 and E18.5 stages. Besides, the results of co-location of EGFPMterfd2 fusion protein indicated that Mterfd2 was targeted to the mitochondria. Collectively, these data suggested that Mterfd2 showed a dynamic expression pattern during embryogenesis. It might play an important role in the organ differentiation which was probably resulted from its role in the mitochondrial transcription regulation.
\end{abstract}

Key words: Mterfd2, expression profile, in situ hybridization, northern blot, immunocytochemistry

\section{INTRODUCTION}

Mitochondrion is the metabolic control organelle in which oxidative phosphorylation takes place. Besides, it plays pleiotropic roles in numerous regulatory and developmental processes, such as apoptosis, senescence and so on (Chan, 2006; Kuo et al., 2009). Mitochondrial dysfunctions contribute to many neurological disorders and metabolic diseases, including Parkinson's disease, MELAS syndrome and diabetes (Mandemakers et al., 2007; Tan et al., 2009; Yoon et al., 2010). Thus, insights into the mitochondrial regulation would give light on the regulation in developmental process and probably on clinical therapy.

The mitochondrion is a semi-autonomic organelle which is widely accepted as developping from an $\alpha$-proteobacterium

Edited by Toshihiko Shiroishi

* Corresponding author. E-mail: Kigo@hit.edu.cn
(Gray et al., 1999). The regulation of mitochondrial at transcription level is dual which results from its genetic origin and is controlled at both initiation and termination level (Asin-Cayuela and Gustafsson, 2007; Scarpulla, 2008). Three proteins, the mitochondrial RNA polymerase (POLRMT), the mitochondrial transcription factor A (TFAM), and the mitochondrial transcription factor B1 or B2 (TFB1M or TFB2M) were reported to be sufficient to initiate mtDNA transcription in vitro (Falkenberg et al., 2002). However, the detailed mechanisms are not well understood despite that most of the components in mitochondrial initiation are well defined. Recent years, a consistent amount of studies have been focused on a nucleus encoded novel protein family-the mitochondrial transcription termination factors (MTERFs).

The MTERFs family is a wide spread protein family which is identified in metazoan and plants, which consists of 4 subfamilies named MTERF1 through MTERF4 (Linder et al., 2005). MTERF1 (mTERF) is the founder 
and also the most well characterized as one of the MTERFs. MTERF1 was firstly purified from human mitochondrial lysate and identified to be a mitochondrial transcription terminator. It promotes transcription termination by binding to a 28-base pair region within the tRNA $^{\text {Leu (UUR) }}$ gene at the position immediately adjacent and downstream of the 16S rRNA gene (Kruse et al., 1989). Through bioinformatics tools, its paralogues like sea urchin $m t D B P$, Drosophila DmTTF, Chlamydomonas reinhardtii Moc1, Mus musculus $m$ TERF, Homo sapiens MTERF2 (mterfd3, mTERFL) and MTERF3 (mterfd1) (Chen et al., 2005; Fernandez-Silva et al., 2001; Li et al., 2005; Roberti et al., 2003, 2006; Schonfeld et al., 2004) with similar structure were found, divided into four subfamilies, and then further characterized. The salient feature of proteins in MTERF family is the containing of variable number of "mterf" motifs of about 30 amino acids in length. This feature is already verified in several MTERF family proteins, for example, Hs-mTERF, PlmtDBP and Dm-DmTTF (Daga et al., 1993; FernandezSilva et al., 2001; Roberti et al., 2003). MTERF2 is found to be a nucleoid component in mammalian and binds to the heavy strand promoter (HSP) region of the mtDNA (Wenz et al., 2009). MTERF3 function as a negative regulator in mammalian mtDNA transcription (Park et al., 2007).

Retrospective studies of three other subfamilies mainly focused on their role in mitochondria transcription regulation. To date, little information of the gene expression profile was reported, especially on the embryonic stage. Moreover, barely any information was elucidated about MTERF4 subfamily despite the fact that a consistent amount of studies has been poured on other subfamilies. With the aim to shed light on the MTERF4 subfamily in mouse, we characterized the expression profile of mouse Mterfd2 during development and its protein subcellular localization.

\section{MATERIALS AND METHODS}

Bioinformatics Protein architecture analysis of the "mTERF motifs" was carried out by taking advantage of the Web tool SMART in Normal mode (http://smart.emblheidelberg.de/). Multiple alignments of mterf domains were performed with DNAMAN 6.0.40 Edition and subsequently processed with Adobe Illustrator CS2. Subcellular localization of Mm-Mterfd2 was predicted using MitoProt II- v1.101 (Claros and Vincens, 1996), TargetP (Emanuelsson et al., 2000), and PSORT (Nakai and Horton, 1999).

Animals and preparation of embryos BALB/cByJ male and female mice were obtained from Beijing Laboratory Animal Research Center (Silaike, Beijing, China). Four animals per cage were fed at the maximum in a $12 \mathrm{~h}$ light/dark cycle controlled chamber (FengShi, Suzhou, China) at room temperature and supplied with commercial feed and tap water with a drop of $\mathrm{HCl}$ per litter. All the experiments conducted with accordance to the "Rules for experiments' animals" published by Chinese Government (Beijing, China). The presence of a vaginal plug on the next morning was counted as 0.5 day post conception (E0.5).

RNA and first strand cDNA synthesis Total RNA was isolated from the embryos and organs according to the standard protocols of Trizol Reagent (Invitrogen, San Diego, USA). The cDNAs were synthesized using a Superscript $^{\mathrm{TM}}$ III RNase $\mathrm{H}^{-}$Reverse Transcriptase kit (Invitrogen); all the experiment procedures were performed according to the manufacturer's protocol.

In situ hybridization (ISH) For the whole mount ISH analysis, embryos were fixed in 4\% paraformaldehyde with phosphate buffered saline (PBS) and incubated at $4^{\circ} \mathrm{C}$ overnight. For tissue ISH, the fixed embryos were dehydrated and subsequently drenched completely in xylene and eventually embedded in paraffin. Sections $(10 \mu \mathrm{m})$ of the embryos were sliced with a microtome (YAMATO, RV-240, Japan) and then adhered on the slides filmed with polylysine. ISH was performed according to the standard protocol described previously (Breitschopf et al., 1992). Digoxigenin (DIG)-UTP labeled RNA probe was synthesized using a DIG RNA labeling kit (Roche, Mannheim, Germany). Forward primer (5'-gttgttcttcgettggtc-3') and reverse primer (5'-cacccctcatttggtttg-3'), plasmid pBluescript II KS (+) (Stratagene, California, USA) were used to generate template for RNA synthesis. All the experiment procedures were performed according to the manufacturer's protocol.

Quantitative Real Time PCR (qRT-PCR) analysis The qRT-PCR was performed by ABI PRISM 7500 RealTime PCR System (ABI, California, USA) with a Prefect Real Time SYBR Premix Ex Taq ${ }^{\mathrm{TM}}$ Kit (TaKaRa, Tokyo, Japan). Primers (forward 5'-tgaagaggcgttccagttac-3' and reverse 5 '-gaacaagcacttctcctga-3') were used. The $\beta$ actin gene was used as control. The primers of $\beta$-actin were forward: 5'-taccacaggcattgtgatggact-3', and reverse: 5'-ttgatgtcacgcacgatttccet-3'.

Northern blot analysis The concentration and purity of the total RNA was evaluated by NanoDrop 2000c (Thermo, Massachusetts, USA). $2 \mu \mathrm{g}$ mRNA was separated through $1 \%$ formaldehyde gels electrophoresis and subsequently blotted onto nylon membrane (Amersham, USA) and subsequently crosslink by exposing to ultraviolet ray $(120 \mathrm{~mJ})$. All the procedures were performed according to the manufacturer's instructions (Roche). $G A P D H$ was used as a loading control. 
Sub-cellular localization of Mterfd2 The mouse Mterfd2 fragment lacking its natural stop codon was amplified from E15.5 mouse brain cDNA using primers forward (5'-tactcactegagatggettcgctggga aggcaggttc-3') and reverse (5'-tactcactgcagcagtagctcctcctcttcct-3'). "ctcgag" and "ctgcag" refer to the restriction sites recognized by XhoI and PstI respectively. "atg" highlights the transcription start site. This PCR product was digested by $X h o \mathrm{I}$ and Pst I, and subsequently cloned into the expression vector pEGFP-N1 (Clontech, USA). The resultant construct, designed as pEGFP-N1-Mterfd2, was sequencing certified and then transiently transfected into NIH3T3 cells using GeneJuice ${ }^{\circledast}$ Transfection Reagent (Merck, Darmstadt, Germany) according to manufacturer's protocol. The pEGFP-N1 vector was transfected in parallel as a control. Transfected cells were grown in

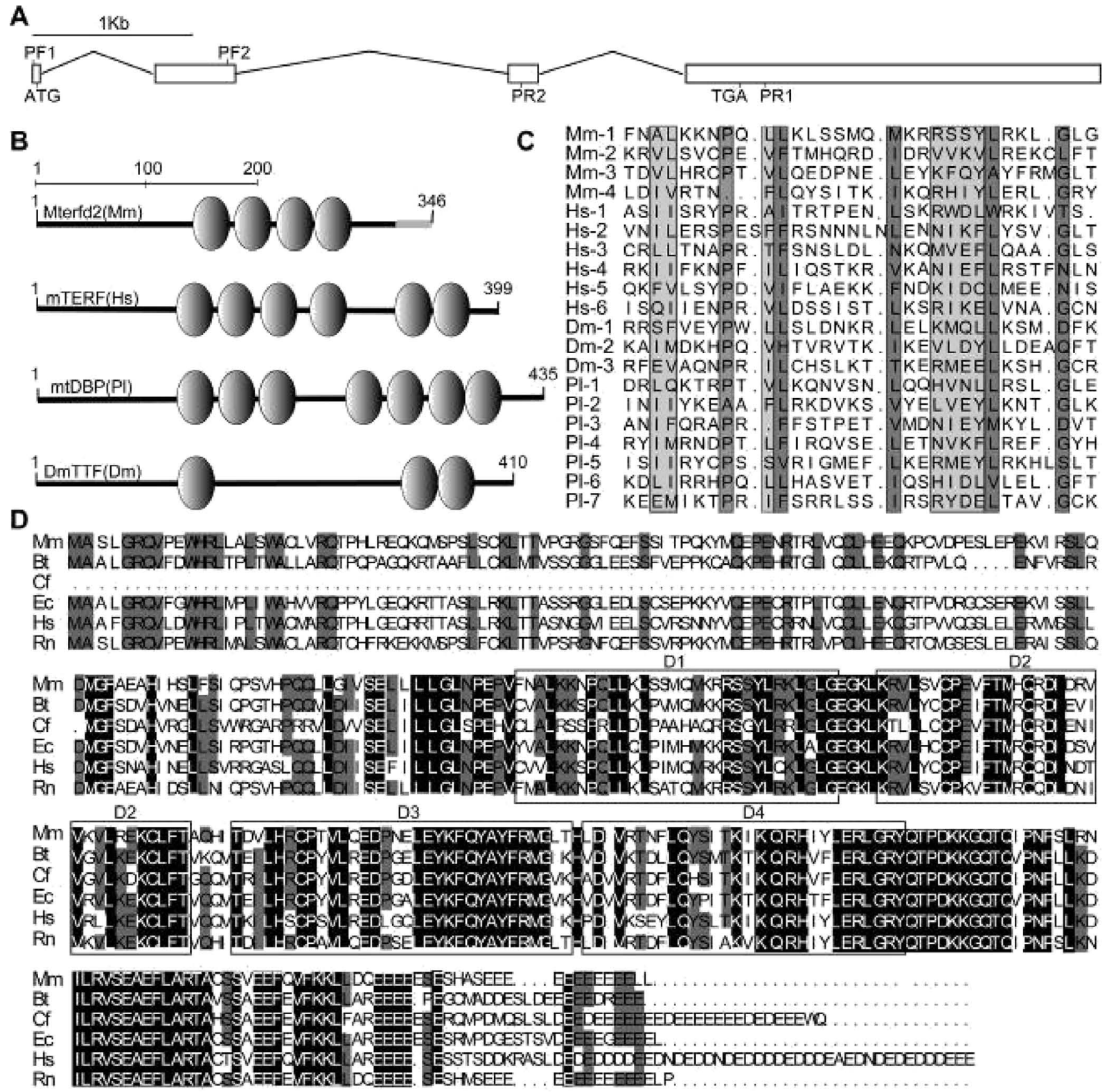

Fig. 1. Bioinformation of Mterfd2. (A) Genomic organization of Mterfd2. The rectangles represent the exons while the broken lines show the introns. ATG and TGA indicate the translation start codon and the termination codon. PF1 and PR1 is the forward and reverse primer used for the generation of the RNA probe, respectively. PF2 and PR2 are for qRT-PCR. (B) Architecture of Mterfd2 and three other well characterized proteins of the mterf family. Black lines show the amino acid chains and grey ellipses indicate mterf motifs of the proteins, respectively. The grey line of the Mterfd2 (Mm) is a coiled-coil region on the C terminal. Specific abbreviations used are Mm (M. musculus), Hs (H. sapiens), Pl (Paracentrotus lividus), Dm (Drosophila melanogaster) respectively. (C) Multiple alignments of the mterf motifs in (B). The motif numbers of the species increase from the $\mathrm{N}$ terminal to the $\mathrm{C}$ terminal. (D) Multiple alignments of the Mterfd2. D1 to D4 highlights the four mterf motifs. New specific abbreviations used are Bt (Bos taurus), Cf (Canis familiaris), Ec (Equus caballus), Rn (Rattus norvegicus), others are identical to (b). 
DMEM supplemented with 5\% FBS for $48 \mathrm{~h}$. The chamber slides were then incubated in regulator medium with $100 \mathrm{mmol} / \mathrm{L}$ MitoTracker Red CMXRos (Invitrogene) for $40 \mathrm{~min}$. The chamber slides were then viewed under a Zeiss confocal fluorescence microscope (LSM510 META, Zeiss).

\section{RESULTS}

Bioinformation of the Mterfd2 The mouse Mterfd2 gene spans $6.7 \mathrm{~Kb}$ on chromosome 1 and consists of four exons which encode a protein of 346 amino acids (Fig. 1A) with a molecular mass of $40.2 \mathrm{kDa}$ and an estimated isoelectric point $(\mathrm{pI})$ value of 6.2. The mature protein is about $37.3 \mathrm{kDa}$ and has a $\mathrm{pI}$ of 5.9 . Similarly to the structure of other well characterized proteins in the family, Mterfd2 exhibits four typical "mterf" motifs (Fig. 1B). Multiple alignment of the amino acid sequence within the motif revealed that the amino acid sequence were highly conserved in the presumptive functional positions, namely a proline residue at position 8 , a leucine at position 11, 18, 25 that can be substituted for another
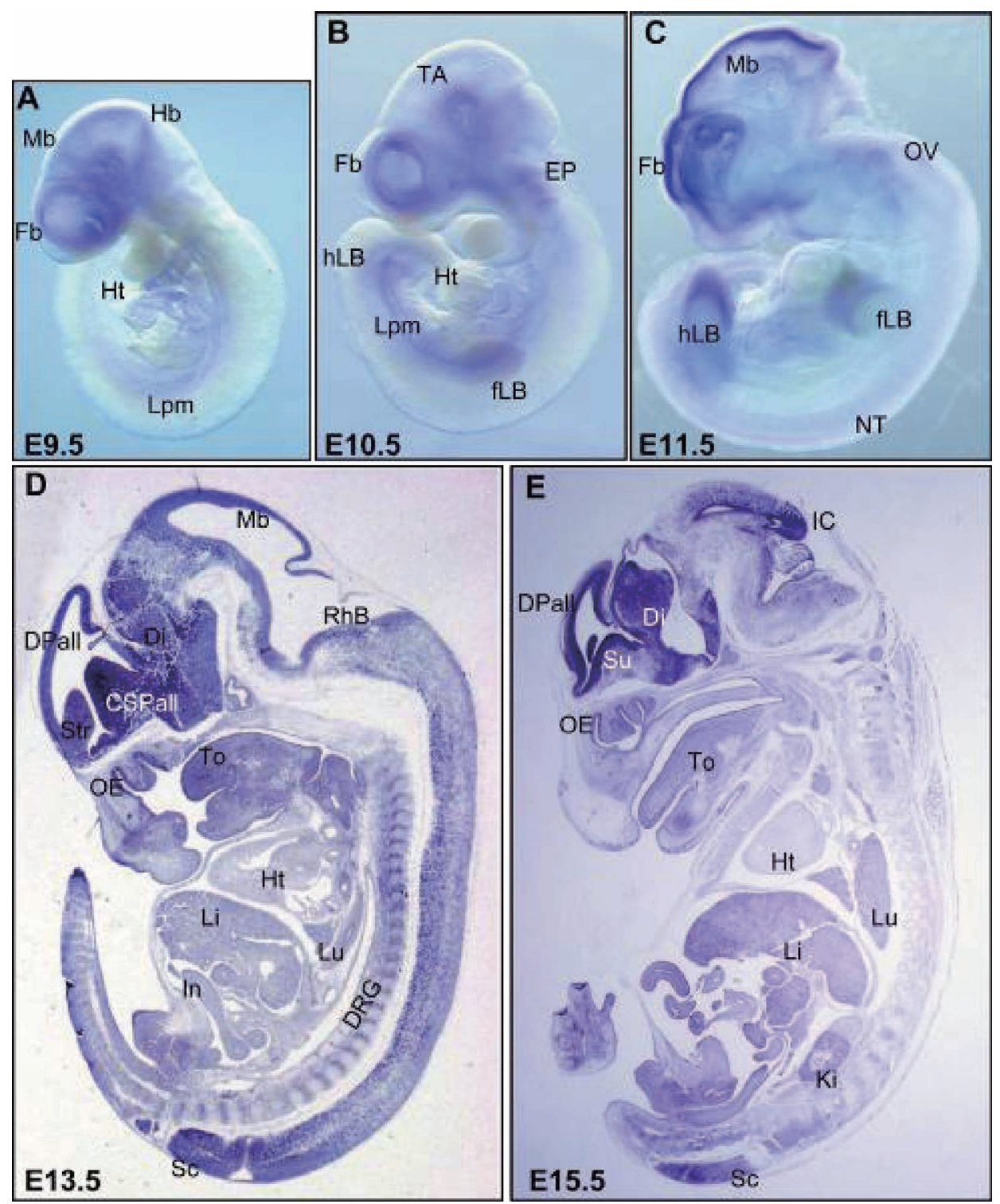

Fig. 2. Mterfd2 expression analysis during mouse development by ISH. ISH were carried out at embryonic stage E9.5 (A) , E10.5 (B) and E11.5 (C) with whole mount mouse embryos, E13.5 (D) and E15.5 (E) with sagittal sections of mouse embryos. CSPall: central subpallium, Di: diencephalon, Dpall: dorsal pallium, DRG: dorsal root ganglia, EP: ear pinna, Fb: forebrain, fLB: fore limb bud, Hb: hindbrain, hLB: hind limb bud, Ht: heart, IC: inferior colliculus, In: intestine, Ki: kidney, Li: liver, Lpm: lateral plate mesoderm, Lu: lung, Mb: midbrain, NT: neural tube, OE: olfactory epithelium, OV: otic vesicle, RhB: roof plate of the hindbrain, Sc: spinal cord, Str: striatum, Su: subpallium, TA: trigeminal area, To: tongue. 
hydrophobic amino acid, a glutamic acid in preference at position 29 (Fig. 1C). To verify the conservation state of meterfd2, we performed multiple alignments of the amino acid residues among species including mouse, cattle, dogs, horse, rats and human beings (Fig. 1D). The results showed that Mterfd2 was highly conserved among mammals, especially in the "mterf" motifs and the C-terminal tail.

In situ hybridization of Mterfd2 To characterize the mouse Mterfd2 expression in embryonic development, whole mount ISH (WISH) was carried out on E9.5 through E11.5 embryos and section ISH (SISH) in E13.5 and E15.5 embryos. The expression of Mterfd2 emerged from the brain, lateral plate mesoderm and heart at E9.5 (Fig. 2A). At E10.5, Mterfd2 expression was found in forebrain, ear pinna, trigeminal area, limbbuds, lateral plate mesoderm and heart (Fig. 2B). At E11.5, Mterfd2 expression was abundant in forebrain, midbrain and limb buds; very weak signals could be detected in the otic vesicle and neural tube (Fig. 2C). At E13.5, Mterfd2 was highly expressed in the brain, spinal cord, dorsal root ganglia, olfactory epithelium and tongue (Fig. 2D). At E15.5, Mterfd2 was highly expressed in the forebrain, midbrain, spinal cord, tong, lung, liver and kidney (Fig. 2E). These data showed that Mterfd2 was comprehensively expressed. As a whole, Mterfd2 shows a persistent and dynamic expression pattern in the early embryonic development stage E9.5 through E15.5.
qRT-PCR and Northern blot analysis of Mterfd2 To confirm the ubiquitous expression of Mterfd2 in E13.5 and E15.5 tissue ISH and whether this phenomenon was common during late embryogenesis, we carried out qRTPCR in multiple tissues obtained from E12.5, E15.5 and

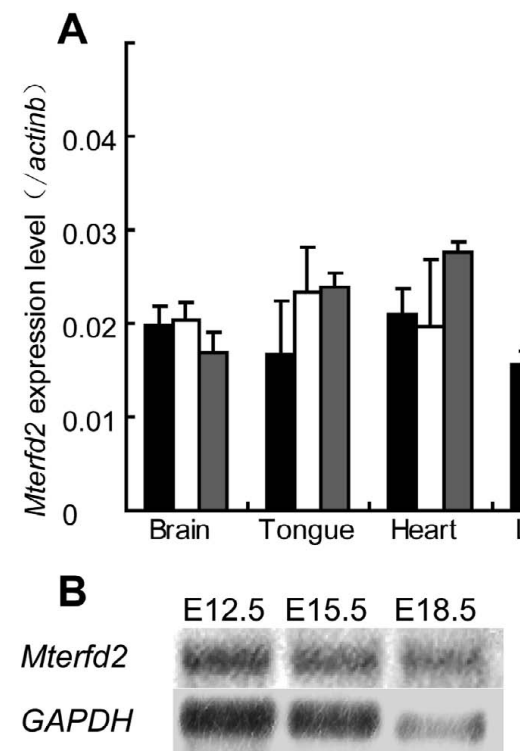

Fig. 3. Mterfd2 expression analysis during embryogenesis. (A) qRT-PCR analysis of Mterfd2. qRT-PCR analysis was performed in six tissues at embryonic stage E12.5, E15.5 and E18.5. Data were tripled at least and presented as the mean with standard deviation as the bar. (B) Northern blot analysis of Mterfd2 by using mRNA isolated from E12.5, E15.5 and E18.5 embryos.

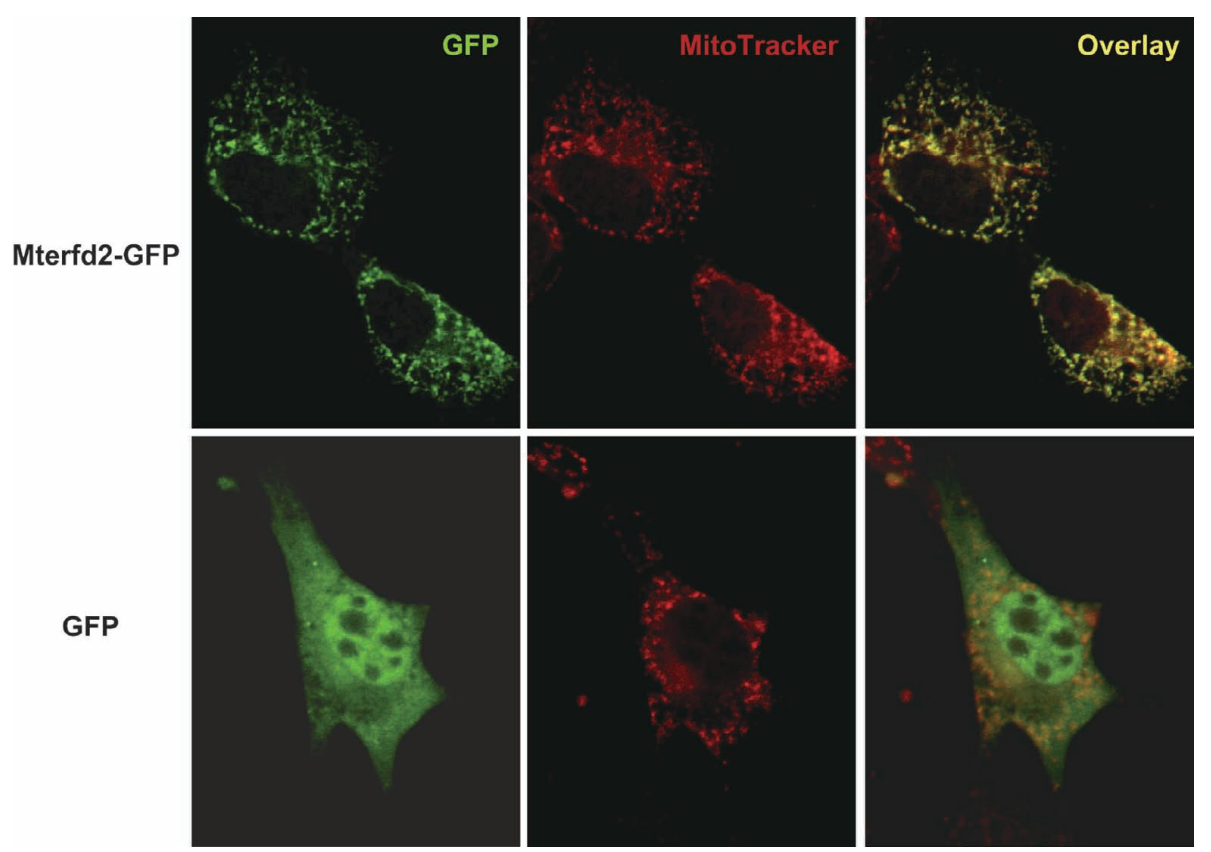

Fig. 4. Sub-cellular localization of mouse Mterfd2 in NIH3T3 cells. Reconstructed plasmid pEGFP-N1Mterfd2 (upper panel) or pEGFP-N1 vector (down panel) were transiently transfected into NIH3T3 cells. The mitochondria were stained in red with CMXRos. The green signals indicate the GFP or Mterfd2-GFP fusion protein. 
E18.5 embryos. The results showed that Mterfd2 was expressed in all tested tissues (Fig. 3A). To further investigate the expression characteristic of Mterfd2, we performed northern blot on mRNAs extracted from E12.5, E15.5 and E18.5 embryos. The results showed that Mterfd 2 was persistently expressed in tested stages, with an up regulation in E18.5 (Fig. 3B). This data further certificated the ubiquitous expression of Mterfd2 during late embryogenesis.

Sub-cellular localization of Mterfd2 Firstly, we used three online software programs to predict sub-cellular localization of the mouse Mterfd2 protein, MitoProt II v1.101 (67\%), TargetP (56.3\%), and PSORT (82\%), with a cleavage side between amino acid residue 25 and 26 , suggesting a high probability of mitochondrial localization.

To get direct evidence for this prediction, we transiently transfected NIH3T3 cell with reconstructed plasmids pEGFP-N1-Mterfd2 which encodes a fusion protein consisting of Mterfd2 fused at it carboxyl terminus to enhanced green fluorescent protein (Mterfd2-EGFP). At the same time, the pEGFP-N1 vector was transfected as a control. The intracellular localization of Mterfd2EGFP overlaps with mitochondria labeled with the fluorophore mitotracker while the green fluorescence in the control cells distributed throughout the cell (Fig. 4).

\section{DISCUSSION}

In this report, we characterized a new component of the MTERF4 subfamily, Mm-Mterfd2, which contains 4 mterf motifs unique to MTERF family. The conservation analysis of Mterfd2 reveals that this protein is highly conserved among mammals, which might be indicative of an evolutionarily conserved function. Differently from other components in the family, Mm-Mterfd2 had a 31 amino acids leucine rich coiled-coil region in the $\mathrm{C}$-terminal tail which is highly conserved among mammals. It is reported that coiled-coil structure plays an important role in protein-protein interaction. For example, in a study concerns Sir4p, it is reported that the C-terminal coiledcoil mediated dimerization played an essential role in silent chromatin at telomeric and silent mating type loci (Murphy et al., 2003). Another research on the yeast transcriptional activator GCN4 showed that coiled-coil dimerization can interact with DNA (Ellenberger et al., 1992). Thus, Mterfd 2 might interact with other proteins through the C-terminal domain.

As part of the research, we focus on the expression profile of Mterfd2 by ISH, qRT-PCR and Northern blot. Our data demonstrated that Mterfd2 is dynamically expressed during embryogenesis. It emerged from the brain, lateral plate mesoderm, and the heart and gradually extended to the whole tissue. These data are consistent with the result that $M$ terfd 2 encoded a mitochondria related protein, and it probably results from the participation in the mitochondrial transcription termination process.

Previous studies have verified the pronounced role of mitochondria dysfunction in neurological disorders and metabolic diseases, including Parkinson's disease, MELAS syndrome and diabetes (Mandemakers et al., 2007; Tan et al., 2009; Yoon et al., 2010). Thus, we paid special attention to the Mterfd2 expression in the nervous system, especially the brain. Our results demonstrated that Mterfd2 expression region became gradually restricted, from the whole brain expression at E9.5 to the highly restricted expression in the pallium of the forebrain and inferior colliculus of the midbrain (E15.5). These results might reflect the dynamic regulation of the Mterfd2 in the brain. This data is supported by the down regulation of $M$ terfd2 in the brain by qRTPCR. Besides, Mterfd2 expression in the spinal cord emerged from E11.5 mouse neural tube. It was quite abundant in the spinal cord at E13.5 and E15.5 stages. It might be reasonable that Mterfd 2 has a role in cognition since the expression was persistently high in the pallium and spinal cord.

Moreover, WISH results showed that Mterfd2 was expressed in the lateral plate mesoderm. At the later embryogenesis stage (E13.5 and E15.5), Mterfd2 was ubiquitously expressed in the main organ and other supportive tissues. These data suggested that Mterfd2 might have multiple roles in organogenesis.

Since the founder of the MTERF family, Hs-mTERF is purified from mitochondrial lysate (Kruse et al., 1989), Hs-MTERF2 ( $m$ TERFL) and MTERF3 and Mm-mTERF are all found to be localized in the same organelle (Chen et al., 2005; Li et al., 2005; Park et al., 2007). It is highly possible that their paralogue Mterfd2 is also targeted to mitochondria. Our result clearly demonstrated this possibility.

Recent reports suggest that mitochondrial regulation is rather complicated and it might be carried out by a complex. Proteins like Hs-mTERF, Pl-mtDBP and DmDmTTF have been shown to participate in the regulation of transcription termination (Daga et al., 1993; FernandezSilva et al., 2001; Kruse et al., 1989; Roberti et al., 2003). The significant protein sequence similarity between mTERF and Mterfd2, as well as the same subcellular localization of these proteins, predicts the important role of Mterfd2 in the modulation of mitochondria transcription. As our study showed that Mterfd2 is targeted to mitochondria, it is predictable that Mterfd2 might execute its transcription regulation function by binding to the regulation elements of the mtDNA, probably the HSP and transcription initiating region. Previous studies have showed that MTERF1 and MTERF2 and MTERF3 co-immunoprecipitate with each other in the HSP region (Wenz et al., 2009). Based on the unique 
structure and the same sub-cellular localization, Mterfd2 may potentially bind to the HSP region and play a central role in coordinating the complex orientation. It is intriguing to us whether and how these factors coordinate with each other in their performance.

This work is financially supported by Ministry of Education Scholarship (Grant No. GFEQ24403001), State Key Laboratory of Urban Water Resource and Environment (Grant No. 2010TS05), Harbin Institute of Technology Introduction of Scientific Research Talents Activation Fee (Grant No. GFQQ18600015), and Heilongjiang Province Technological Project Program Returning Foundation (Grant No. LC08C05).

\section{REFERENCES}

Asin-Cayuela, J., and Gustafsson, C. M. (2007) Mitochondrial transcription and its regulation in mammalian cells. Trends Biochem. Sci. 32, 111-117.

Breitschopf, H., Suchanek, G., Gould, R. M., Colman, D. R., and Lassmann, H. (1992) In situ hybridization with digoxigeninlabeled probes: sensitive and reliable detection method applied to myelinating rat brain. Acta Neuropathol. 84, 581-587.

Chan, D. C. (2006) Mitochondria: dynamic organelles in disease, aging, and development. Cell 125, 1241-1252.

Chen, Y., Zhou, G., Yu, M., He, Y., Tang, W., Lai, J., He, J., Liu, W., and Tan, D. (2005) Cloning and functional analysis of human mTERFL encoding a novel mitochondrial transcription termination factor-like protein. Biochem. Biophys. Res. Commun. 337, 1112-1118.

Claros, M. G., and Vincens, P. (1996) Computational method to predict mitochondrially imported proteins and their targeting sequences. Eur. J. Biochem. 241, 779-786.

Daga, A., Micol, V., Hess, D., Aebersold, R., and Attardi, G. (1993) Molecular characterization of the transcription termination factor from human mitochondria. J. Biol. Chem. 268, 8123-8130.

Ellenberger, T. E., Brandl, C. J., Struhl, K., and Harrison, S. C. (1992) The GCN4 basic region leucine zipper binds DNA as a dimer of uninterrupted alpha helices: crystal structure of the protein-DNA complex. Cell 71, 1223-1237.

Emanuelsson, O., Nielsen, H., Brunak, S., and von Heijne, G. (2000) Predicting subcellular localization of proteins based on their N-terminal amino acid sequence. J. Mol. Biol. 300, 1005-1016.

Falkenberg, M., Gaspari, M., Rantanen, A., Trifunovic, A., Larsson, N. G., and Gustafsson, C. M. (2002) Mitochondrial transcription factors $\mathrm{B} 1$ and $\mathrm{B} 2$ activate transcription of human mtDNA. Nat. Genet. 31, 289-294.

Fernandez-Silva, P., Polosa, P. L., Roberti, M., Di Ponzio, B., Gadaleta, M. N., Montoya, J., and Cantatore, P. (2001) Sea urchin $\mathrm{mtDBP}$ is a two-faced transcription termination factor with a biased polarity depending on the RNA polymerase. Nucleic Acids Res. 29, 4736-4743.

Gray, M. W., Burger, G., and Lang, B. F. (1999) Mitochondrial evolution. Science 283, 1476-1481.

Kruse, B., Narasimhan, N., and Attardi, G. (1989) Termination of transcription in human mitochondria: identification and purification of a DNA binding protein factor that promotes termination. Cell 58, 391-397.

Kuo, H. M., Tsai, H. C., Lin, Y. L., Yang, J. S., Huang, A. C., Yang, M. D., Hsu, S. C., Chung, M. C., Gibson Wood, W., and Chung, J. G. (2009) Mitochondrial-dependent caspase activation pathway is involved in baicalein-induced apoptosis in human hepatoma J5 cells. Int. J. Oncol. 35, 717-724.

Li, X., Zhang, L. S., and Guan, M. X. (2005) Cloning and characterization of mouse mTERF encoding a mitochondrial transcriptional termination factor. Biochem. Biophys. Res. Commun. 326, 505-510.

Linder, T., Park, C. B., Asin-Cayuela, J., Pellegrini, M., Larsson, N. G., Falkenberg, M., Samuelsson, T., and Gustafsson, C. M. (2005) A family of putative transcription termination factors shared amongst metazoans and plants. Curr. Genet. 48, 265-269.

Mandemakers, W., Morais, V. A., and De Strooper, B. (2007) A cell biological perspective on mitochondrial dysfunction in Parkinson disease and other neurodegenerative diseases. J. Cell Sci. 120, 1707-1716.

Murphy, G. A., Spedale, E. J., Powell, S. T., Pillus, L., Schultz, S. C., and Chen, L. (2003) The Sir4 C-terminal coiled coil is required for telomeric and mating type silencing in Saccharomyces cerevisiae. J. Mol. Biol. 334, 769-780.

Nakai, K., and Horton, P. (1999) PSORT: a program for detecting sorting signals in proteins and predicting their subcellular localization. Trends Biochem. Sci. 24, 34-36.

Park, C. B., Asin-Cayuela, J., Camara, Y., Shi, Y., Pellegrini, M., Gaspari, M., Wibom, R., Hultenby, K., Erdjument-Bromage, H., Tempst, P., Falkenberg, M., Gustafsson, C. M., and Larsson, N. G. (2007) MTERF3 is a negative regulator of mammalian mtDNA transcription. Cell 130, 273-285.

Roberti, M., Bruni, F., Polosa, P. L., Manzari, C., Gadaleta, M. N., and Cantatore, P. (2006) MTERF3, the most conserved member of the mTERF-family, is a modular factor involved in mitochondrial protein synthesis. Biochim. Biophys. Acta 1757, 1199-1206.

Roberti, M., Polosa, P. L., Bruni, F., Musicco, C., Gadaleta, M. N., and Cantatore, P. (2003) DmTTF, a novel mitochondrial transcription termination factor that recognises two sequences of Drosophila melanogaster mitochondrial DNA Nucleic Acids Res. 31, 1597-1604.

Scarpulla, R. C. (2008) Transcriptional paradigms in mammalian mitochondrial biogenesis and function. Physiol. Rev. 88, 611-638.

Schonfeld, C., Wobbe, L., Borgstadt, R., Kienast, A., Nixon, P. J., and Kruse, O. (2004) The nucleus-encoded protein MOC1 is essential for mitochondrial light acclimation in Chlamydomonas reinhardtii. J. Biol. Chem. 279, 50366-50374.

Tan, T. M., Caputo, C., Medici, F., Pambakian, A. L., Dornhorst, A., Meeran, K., Williams, G. R., and Khoo, B. (2009) MELAS syndrome, diabetes and thyroid disease: the role of mitochondrial oxidative stress. Clin. Endocrinol. (Oxf) 70, 340341.

Wenz, T., Luca, C., Torraco, A., and Moraes, C. T. (2009) mTERF2 regulates oxidative phosphorylation by modulating mtDNA transcription. Cell Metab. 9, 499-511.

Yoon, Y., Galloway, C. A., Jhun, B. S., and Yu, T. (2010) Mitochondrial dynamics in diabetes. Antioxid. Redox Signal. 14, 439-457. 